\title{
Evaluation of the Cult-Dip Plus dip slide method for urinary tract infection
}

\begin{abstract}
J M Blondeau, Y Yaschuk, D Galenzoski, D Hrabok, M Isaacson, L Lee, H Link, L Walshaw
\end{abstract}

results comparable with those obtained using standard plate streaking methods..$^{3-7}$ Dip slides that incorporate substrates for the detection of bacterial enzymes could result in the early identification of uropathogens. Kilian and Bulow $^{8}$ showed that $E$ coli was one of the few bacteria to produce the enzyme B-glucuronidase. Hydrolysis of 4-methylumbelliferylB-D-glucuronide (MUG) by B-glucuronidase produces 4-methylumbelliferon-a product which fluoresces under ultraviolet light.

Cult-Dip Plus is a new urine dip slide consisting of Brolacin (cystine, lactose, electrolyte deficient (CLED)) and MacConkey agar, each containing MUG. We evaluated the Cult-Dip Plus for recovery of uropathogens and the MUG test for rapid identification of $E$ coli.

\section{Methods}

The MUG test is based on detection of fluorescence from release of 4-methylumbelliferon following hydrolysis of the parent compound by B-glucuronidase. Cult-Dip Plus was supplied by BDH (Toronto, Ontario, Canada). Blood and CLED agars were purchased from PML Microbiologicals (Edmonton, Alberta, Canada)

We prospectively analysed 1022 urine samples from different patients submitted over a two month period. The semiquantitative plate culture method described by Clarridge et al ${ }^{9}$ was used as the reference method for determining bacteriuria. For routine culture, $0.001 \mathrm{ml}$ urine was delivered to each blood and CLED agar plate using a calibrated disposable loop (Simport, Quebec, Canada). This method detects $\geq 1000$ colony forming units/ $\mathrm{ml}(\mathrm{cfu} / \mathrm{ml})$

Following this, the Cult-Dip Plus dip slide was inoculated by either immersing the slide in the urine (minimum $30 \mathrm{ml}$ required) or when the volume of urine was such that the paddle could not be immersed, by flooding both sides of the slide with urine using a sterile disposable pasteur pipette $(5 \mathrm{ml})$. This method detects $100 \mathrm{cfu} / \mathrm{ml}$. Colony counts of 1-10 colonies detected by Cult-Dip Plus were considered negative so as to standardise the colony count comparisons between both methods. After inoculation, culture plates/dip slides were incubated for $18-24$ hours at $35-37^{\circ} \mathrm{C}$. for determining the causative organism and because it affects the appropriate selection or continuation, or both, of antimicrobial therapy.

Urine dip slides are immersible plastic paddles containing agar medium on each side. Dip slides have previously been shown to be cost-effective, easy to use devices that provide
IDENTIFICATION OF MICRO-ORGANISMS

Typical lactose fermenting colonies resembling $E$ coli were identified by using the Bactident $E$ coli test (BDH, Toronto, Ontario, Canada), a 
Table 1 Comparison of 1022 urine specimens isolated by Cult-Dip Plus and routine culture

\begin{tabular}{llll}
\hline & Both methods & Cult-Dip Plus & Routine culture \\
\hline Negative $^{+}$ & 761 & 24 & 16 \\
Positive* $^{*}$ & 181 & 16 & 24
\end{tabular}

* Pure isolates in quantities greater than $10000 \mathrm{cfu} / \mathrm{ml}$.

+ Includes those with no visible growth and those with two or more species of micro-organism (mixed)

Table 2 Frequency of uropathogens isolated by Cult-Dip Plus and routine culture

\begin{tabular}{llll}
\hline Micro-organism & Both methods & Cult-Dip Plus & Routine method \\
\hline Gram negative bacilli & 131 & 13 & 11 \\
Gram positive cocci & 38 & 3 & 13 \\
Yeast & 12 & - & - \\
All genera & 181 & 16 & 24 \\
\hline
\end{tabular}

Table 3 Summary of uropathogens MUG test positive by either Cult-Dip Plus, Bacident, or both

\begin{tabular}{lllll}
\hline & \multicolumn{2}{l}{$\begin{array}{l}\text { Cult-Dip Plus Isolates } \\
\text { No. positivelno. tested (\%)) }\end{array}$} & & $\begin{array}{l}\text { Routine culture } \\
\text { No. positivelno. tested (\%)) }\end{array}$ \\
\cline { 2 - 3 } Micro-organism & Cult-Dip Plus & Bactident & & Bactident \\
\hline E coli & $88 / 96(92)$ & $64 / 68(94)$ & & $70 / 77(91)$ \\
K pneumoniae & $4 / 16(25)$ & NT & & NT \\
P mirabilis & $2 / 10(20)$ & NT & NT \\
\hline
\end{tabular}

NT $=$ not tested.
Compared with the calibrated loop method Cult-Dip Plus had a sensitivity of $88.3 \%$, a specificity of $98.0 \%$, a positive predictive value of $91.9 \%$, and a negative predictive value of $97 \cdot 1 \%$ (table 1 ).

There were 221 significant organisms identified. Table 2 shows the frequency of uropathogens detected by either Cult-Dip Plus, routine culture or both. As expected, $E$ coli was the most frequently recovered pathogen. In all cases where a significant uropathogen was recovered by either Cult-Dip Plus or by routine culture but not by both, the corresponding method had mixed organisms and was not investigated further.

Table 3 shows the results of the MUG test for identification of $E$ coli. Of the $E$ coli isolates, 92\% were MUG test positive compared with $25 \%$ of $K$ pneumoniae and $20 \%$ of $P$ mirabilis isolates recovered by Cult-Dip Plus. None of the remaining isolates were MUG test positive.

In total, $105 E$ coli isolates were recovered as significant uropathogens. Of these, 88 were recovered by both methods, eight by Cult-Dip Plus only and nine by routine culture only. Of the $99 E$ coli isolates recovered by Cult-Dip Plus, 96 underwent a MUG test (three were not viewed for fluorescence and were not analysed further) (table 3). Of the 96 tested, 88 (92\%) were MUG test positive. Detection of fluorescence was similar for both media on the dip slide. Thirty one of the 99 isolates recovered by Cult-Dip Plus were identified by Vitek and 68 were identified by Bactident. Of the 68 isolates tested by Bactident, 64 (94\%) were MUG test positive. Of the eight $E$ coli isolates detected by Cult-Dip Plus only and which were MUG test negative, five were tested by Bactident and three were MUG test positive (see discussion). Overall, the Cult-Dip Plus MUG test had a sensitivity of $91.6 \%$ and a specificity of $95 \cdot 2 \%$ and a positive predictive value of $93.6 \%$ for identifying $E$ coli.

Of the $95 E$ coli isolates recovered by routine culture, 77 were tested by Bactident and 70 (91\%) were MUG test positive (table 3 ). Of the 70 MUG test positive isolates, $63(90 \%)$ were also positive by the Cult-Dip Plus MUG test. Of the seven remaining isolates, five were Cult-Dip Plus MUG test negative and the remaining two were untested. Of the 19 routine cultures that the Bactident MUG test were not performed on, 16 were Cult-Dip Plus MUG test positive and three were not tested. $(21.6 \%)$ had significant findings $(>10000 \mathrm{cfu} /$ $\mathrm{ml}$ and pure) by one method or the other; 181 $(81.9 \%)$ were detected by both methods; 24 $(10.9 \%)$ by the routine method only; and 16 $(7 \cdot 2 \%)$ by Cult-Dip Plus only. Table 1 shows the distribution of the data. With regard to insignificant (mixed with two or more species of micro-organisms or $<10000 \mathrm{cfu} / \mathrm{ml}$ ) or negative results (no visible growth), 417 (40.8\%) urine samples were negative by both methods; $344(33.7 \%)$ had mixed or insignificant counts by both methods and of these, $162(15.9 \%)$ had colony counts that differed between both methods (data not shown). Of the urine samples cultured by Cult-Dip Plus, 97 had $<1000$ $\mathrm{cfu} / \mathrm{ml}$; however, these were scored as no growth when the cut off was adjusted to $\geq 1000$ $\mathrm{cfu} / \mathrm{ml}$ for comparison with routine culture.

\section{Discussion}

The dip slide was introduced in $1967^{1011}$ and represented a further development of the dipspoon method. ${ }^{12}$ It was originally seen as an inexpensive, easy to use and convenient system that could be used at the bedside by nonlaboratory personnel and still provide reliable and clinically meaningful results. ${ }^{5}$ Many laboratories use dip slides as the method of choice for detecting uropathogens. Kennon and Soderdahl ${ }^{13}$ showed that the dip slide was a cost-effective alternative to conventional urine culture methodology. 
Rosenberg $e t a l^{7}$ found that the sensitivity, specificity and positive predictive value of dip slide versus routine culture was $98.8 \%, 95.7 \%$ and $97 \cdot 2 \%$, respectively, and $97 \cdot 0 \%, 98.3 \%$ and $98.3 \%$, respectively, for the diaslide urine culture device versus the calibrated loop routine culture method. Our findings of sensitivity, specificity and positive predictive values of $88.3 \%, 98.0 \%$ and $91.9 \%$, respectively, are slightly lower than those reported by Rosenberg et al. This difference could be explained by the differences in the two dip slides used in these studies. Alternatively, it could be explained by the difference in urine specimens used in the evaluation: Rosenberg et al prescreened urine samples for catalase activity in order to increase the proportion of positive cultures tested and we did not. Rosenberg et al state that evaluations using non-screened urine samples should be performed in addition to studies using prescreened urines.

The data showing significant findings detected in urine specimens by one method only (table 1) require further discussion. As previously stated, in all instances where one method (but not both) had significant findings, the corresponding method was scored as having mixed or insignificant findings. Some of these specimens may have been interpreted differently in another laboratory. Given that dip slides sample a larger volume of urine, they probably detect micro-organisms present in lower numbers (that is, contaminants) along with those associated with urinary tract infections. There is no universally accepted protocol for the interpretation of urine cultures. Rather, established guidelines ${ }^{9}$ are widely used; however, interpretation of these guidelines in some clinical situations may vary from one laboratory to another. Therefore, sensitivity and specificity of product evaluations as highlighted here may be different if different interpretative criteria are applied. Laboratories interested in changing their current methodology might best be served by performing evaluations of their own to determine performance of new methodology under their own interpretative criteria.

Ellner and Papachristos ${ }^{3}$ compared urine dip slides with routine culture and found that the urine dip slides gave equivalent results to those obtained by conventional culture. The percentage of specimens from their evaluations with significant (dip slide, culture: 18.6, 15.3), negative $(40.3,31.4)$ and insignificant $(41 \cdot 2$, 43.3) findings was similar to the results from this current evaluation. Additionally, the percentage and overall distribution of the uropathogens detected in this study is similar to that of Ellner and Papachristos.

Our study showed that there were fewer urine samples with no growth and more with mixed or insignificant findings by Cult-Dip Plus than by routine culture. These findings are undoubtedly due to the fact that Cult-Dip Plus can detect $\geq 100 \mathrm{cfu} / \mathrm{ml}$ compared with $\geq 1000 \mathrm{cfu} / \mathrm{ml}$ by routine culture. However, adjusting the cut off for Cult-Dip Plus changes the percentage of specimens with mixed or insignificant findings giving $37.4 \%$ for both Cult-Dip Plus and routine culture.
B-glucuronidase activity in the genera of $E s-$ cherichiae, Salmonella and Shigella was described by Kilian and Bulow in 1976. ${ }^{8}$ Feng and Hartman ${ }^{14}$ and Hansen and Yourassowsky ${ }^{15}$ used B-glucuronidase actively to identify isolates of $E$ coli from water and urine cultures, respectively. Trepeta and Edberg ${ }^{16}$ added the fluorogenic substrate 4-methylumbelliferyl-B-D-glucuronide to agar plates and were then able to detect $E$ coli by ultraviolet light fluorescence of 4-methylumbelliferon following hydrolysis by B-glucuronidase.

Delisle and Ley ${ }^{17}$ and Heizmann $e t a l^{18}$ found that $89.5 \%$ and $90.5 \%$, respectively, of $E$ coli isolates were positive for B-glucuronidase activity. In both of these studies the substrate for detection of B-glucuronidase was incorporated directly into solid media. Our results $(91.6 \%)$ are consistent with their results. It would be unlikely that $100 \%$ of $E$ coli isolates would be MUG test positive as previous reports ${ }^{815}$ indicate that only $94-97 \%$ of isolates were positive for this enzyme.

Our finding of three $E$ coli isolates that were negative for fluorescence (MUG test negative) by Cult-Dip Plus were subsequently MUG test positive by Bactident can be explained by previous studies showing that both $\mathrm{pH}$ and colour change during lactose fermentation can suppress fluorescence. ${ }^{1819}$ We have found that there was considerable variability in both the presence and intensity of fluorescence produced by micro-organisms present on the dipstick. This problem is easily overcome by the addition of $1 \mathrm{~N} \mathrm{NaOH}$ to the colonies of microorganisms. Changing the $\mathrm{pH}$ from acidic to basic intensifies the fluorescence, making detection much easier. It is possible, therefore, that the three $E$ coli isolates that were negative for fluorescence on the Cult-Dip Plus and MUG test positive by Bactident were, in fact, isolates that were falsely negative due to failure to intensify fluorescence by adjusting the $\mathrm{pH}$.

In conclusion, urine dip slides are easy to use, reliable and a cost-effective alternative to routine culture methods for detecting significant bacteriuria. ${ }^{313}$ The Cult-Dip Plus, with the addition of the substrate for detection of B-glucuronidase, is a convenient product for both recovery of uropathogens and the rapid identification of $E$ coli. The MUG test in combination with lactose fermentation and indole formation is reliable for the identification of $E$ coli. Given that $E$ coli is the pathogen recovered most frequently from patients with urinary tract infections, the Cult-Dip Plus will most likely be a cost-effective alternative to other methods currently in use for rapid identification of this micro-organism in urine specimens. We have calculated in our laboratory that of the $754 \mathrm{E}$ coli (data not shown) isolates recovered from urine specimens over a 49 week period, use of Cult-Dip Plus versus our current methodology (plated media and Bactident) would have resulted in a $43 \%$ saving per isolate. This saving may be greater or smaller in other laboratories depending on methodology and volume discounts on products. A further addition of tryptophan to the medium would enable a spot 
indole test to be performed, thereby expanding the utility of this product.

Bacident is a two hour test for identifying $E$ coli. It consists of the MUG test and an indole test. In our laboratory only those micro-organisms with a colonial appearance suggestive of $E$ coli are processed by this method. A spot indole test is performed before using this method. In the results section it is noted that not all $E$ coli isolates recovered by routine culture were tested by Bacident. These isolates would not have been tested because they were either spot indole negative or did not have a typical colonial appearance, or both.

We thank D Leciuk for clerical assistance.

These findings were presented, in part, at the 60th Conjoint Meeting of Infectious Diseases, Toronto, Ontario, Canada, December 2, 1992; Abstract \#T-3

1 Johnson JR, Stamm WE. Urinary tract infections in women: Diagnosis and treatment. Ann Intern Med 1989;111:90617.

2 Harding GKM, Nicolle LE, Ronald AR, Preiksaitis JK, Forward KR, Low DE, et al. How long should catheteracquired urinary tract infection in women be treated? A randomized controlled study. Ann Intern Med 1991;114: 713-19.

3 Ellner PD, Papachristos T. Detection of bacteriuria by dipslide. Routine use in a large general hospital. Am $\mathcal{f}$ Clin Pathol 1975;63:516-21.

4 Gillenwater JY, Gleason CH, Lohr JA, Marion D. Home urine cultures by the dip-strip method: Results in 289 cultures. Pediatrics 1976;58:508-12.
5 McAllister TA. The day of the dipslide. Nephron 1973;11: 123-33.

6 McAllister TA, Arneil GC, Barr W, Kay P. Assessment of plane dipslide quantitation of bacteriuria. Nephron 1973; 11:111-22.

7 Rosenberg M, Berger SA, Barki M, Goldberg S, Fink A, Miskin A. Initial testing of a novel urine culture device. f Clin Microbiol 1992;30:2686-91.

8 Kilian M, Bulow P. Rapid diagnosis of Enterobacteriaceae. I. Detection of bacterial glycosidases. Acta Pathol Microbiol Scand 1976;84:245-51

9 Clarridge JE, Pezzlo MT, Vosti KL. Cumitech 2a 1987. Laboratory diagnosis of urinary tract infections. Weissfeld $\mathrm{AL}$, coordinating ed. Washington DC: American Society for Microbiology, 1987.

10 Cohen SN, Kass EH. A simple method for quantitative urine culture. N Engl f Med 1967;277:176-80.

11 Guttmann D, Naylor GRE. Dipslide: An aid to quantitative urine culture in general practice. BMf 1967;3:343-5.

12 MacKey JP, Sandys GH. Laboratory diagnosis of infections of the urinary tract in general practice by means of a dipinoculum transport medium. BMF 1965;2:1286-8.

13 Kennon WG, Soderdahl DW. Dipslide urine cultures and cost containment. Surg Gynecol Obstet 1982;155:807-8.

14 Feng PCS, Hartman PA. Fluorogenic assays for immediate confirmation of Escherichia coli. Appl Environ Microbiol 1982;43:1320-9.

15 Hansen W, Yourassowsky E. Detection of B-glucuronidase in lactose-fermenting members of the family Enteroin lactose-fermenting members of the family Enterof Clin Microbiol 1984;30:1177-9.

16 Trepeta RW, Edberg SC. Methylumbelliferyl-B-D-glucuronide based medium for rapid isolation and identification of Escherichia coli. $\mathcal{f}$ Clin Microbiol 1984;19. $172-4$.

17 Delisle GJ, Ley A. Rapid detection of Escherichia coli in urine samples by a new chromogenic B-glucuronidase assay. F Clin Microbiol 1989;27:778-9.

18 Heizmann W, Doller PC, Gutbrod B, Werner H. Rapid identification of Escherichia coli by fluorocult media and positive indole reaction. 7 Clin Microbiol 1988;26:2682-4.

19 Edberg SC, Kontnick CM. Comparison of B-glucuronidase based substrate systems for identification of Escherichia coli. F Clin Microbiol 1986;24:368-71. 